\title{
Missing-Row Surface Reconstruction of Ag(110) Induced by Potassium Adsorption
}

\author{
J. W. M. Frenken, ${ }^{(a)}$ R. L. Krans, and J. F. van der Veen \\ FOM-Institute for Atomic and Molecular Physics, \\ 1098 SJ Amsterdam, The Netherlands \\ and \\ E. Holub-Krappe ${ }^{(b)}$ and K. Horn \\ Fritz-Haber-Institut der Max-Planck-Gesellschaft, 1000 Berlin 33, West Germany \\ (Received 25 June 1987)
}

\begin{abstract}
We report a medium-energy ion-scattering study of the $(1 \times 2)$ reconstruction of the $A g(110)$ surface induced by submonolayer amounts of $\mathrm{K}$. A qualitative comparison between ion-scattering data from the clean and $\mathrm{K}$-covered surfaces shows that the $(1 \times 2)$ periodicity is caused by a missing-row reconstruction of the Ag substrate. Computer simulations support this conclusion and provide detailed information on the atomic positions. Our observations support a recent model by Heine and Marks explaining surface relaxation and reconstruction in terms of the occupation of $s-p$ and $d$ electronic levels.
\end{abstract}

PACS numbers: $68.35 . \mathrm{Bs}, 61.80 . \mathrm{Mk}$

The (110) surfaces of $\mathrm{Au}, \mathrm{Pt}$, and $\mathrm{Ir}$ exhibit a $(1 \times 2)$ low-energy electron-diffraction (LEED) pattern. The doubled periodicity observed for these materials along the [001] surface azimuth is caused by a surface reconstruction in which every other [110] surface-atom row is missing. ${ }^{1,2}$ The (110) surfaces of $\mathrm{Ag}, \mathrm{Cu}, \mathrm{Pd}$, and $\mathrm{Ni}$, on the other hand, show a $(1 \times 1)$ LEED pattern and are unreconstructed. ${ }^{3-6}$ Recently it was discovered that the deposition of small amounts of alkali-metal atoms induces a $(1 \times 2)$ LEED pattern also on these surfaces. ${ }^{7-10}$ Since the patterns are induced by alkali-metal atoms with widely differing atomic and ionic radii, an interpretation in terms of an adsorbate $(1 \times 2)$ overlayer is unlikely. Charge donation from the alkali metal to the metal surface has been suggested to drive the reconstruction by alteration of the relative population of $s-p$ and $d$ levels. ${ }^{11}$ Thus the study of the structure of the reconstructed surface may add to the understanding of the relative influence of different electronic levels on the geometric arrangement of surface atoms. The role of alkali-metal atoms as promoters in heterogeneous catalysis ${ }^{12}$ makes the alkali-metal-induced reconstruction also interesting from a practical point of view, since a modification of the substrate structure may well be responsible for an enhancement of the catalytic activity of the metal surface.

Earlier structural studies of $(1 \times 2)$ reconstructed (110) surfaces of $\mathrm{Ag}, \mathrm{Cu}$, and $\mathrm{Pd}$ are conflicting. Helium-atom-diffraction measurements of the Cs/ $\mathrm{Ag}(110)$ system $^{8}$ were found to differ strongly from similar measurements on the missing-row systems $\mathrm{Au}(110)^{13}$ and $\mathrm{Pt}(110) .^{14}$ An ion-scattering study of the $\mathrm{Li} / \mathrm{Cu}(110)$ system, ${ }^{9}$ on the other hand, as well as a LEED study of $\mathrm{Na}$ and $\mathrm{Cs}$ on $\mathrm{Pd}(110),{ }^{6}$ yielded evidence in favor of either missing-row or sawtooth ${ }^{15}$ reconstruction. These reconstruction models and a model with paired rows are depicted in Fig. 1.

In this Letter we present a medium-energy ionshadowing and -blocking ${ }^{16}$ investigation of the $K /$ $\mathrm{Ag}$ (110) system, which allows us to discriminate between different surface-structure models on the basis of purely qualitative arguments. It is demonstrated that alkali-metal adsorption results in the formation of a surface structure of the missing-row type.

The Ag(110) specimen was prepared with standard procedures, ${ }^{4}$ and after cleaning by cycles of ion bombardment and annealing gave rise to a bright $(1 \times 1)$ LEED pattern with low background. The $\mathrm{K}$ depositions (a)

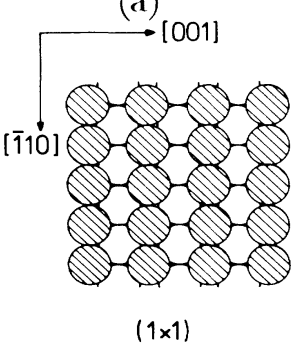

(c)

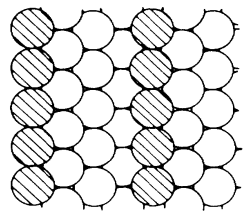

"saw tooth" model (b)

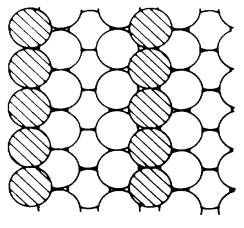

missing rows

(d)

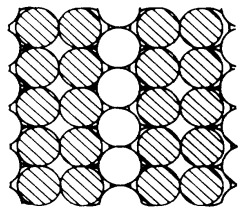

paired rows
FIG. 1. Structure models considered for the $(1 \times 2)$ reconstructed $\mathrm{Ag}(110)$ substrate. Hatched circles denote atoms in the top layer. 
were made as clean as possible, by continuous outgassing of the sources (SAES Getters SpA, Italy) prior to deposition, in a separate UHV chamber. The quality of the $(1 \times 2)$ LEED pattern was greatly improved by subsequent annealing for several minutes at $400 \mathrm{~K}$. K coverages at which a $(1 \times 2)$ LEED pattern was obtained in this study were calibrated with Rutherford backscattering to range from 0.13 to 0.39 monolayer ( 1 monolayer $\equiv 0.85 \times 10^{15}$ atoms $/ \mathrm{cm}^{2}$ ), with the lower coverage corresponding to the highest-quality LEED pattern.

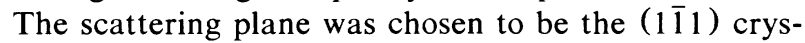
tal plane, which is perpendicular to the (110) surface and runs diagonally across the unreconstructed surface unit cell. A parallel beam of $50.6-\mathrm{keV}$ protons was directed onto the surface along the [101] crystal axis, as shown in Fig. 2. The first atom in each [101] row casts a shadow on the subsequent atoms along the row, thereby strongly reducing the probability for the protons to hit these deeper-lying atoms. A toroidal electrostatic analyzer provided with a position-sensitive channel-plate detector was used to collect spectra of backscattered protons simultaneously over a $20^{\circ}$ angular range. Such a spectrum consists of a "surface peak" with the backscattering signal from the nonshadowed atoms in the surface region, and a low "minimum yield" from the small fraction of nonshadowed subsurface atoms, appearing at

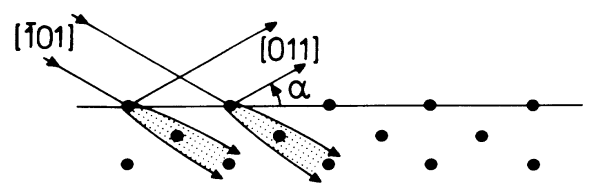

(a)

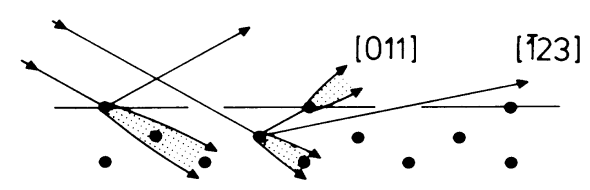

(b)

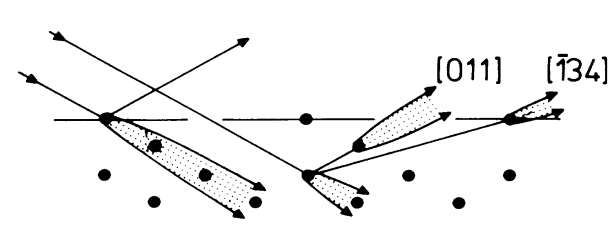

(c)

FIG. 2. Side views of the (1i1) scattering plane, perpendicular to the (110) surface, for (a) an unreconstructed surface, (b) the missing-row model, and (c) the sawtooth model. For the latter model only one of the two possible orientations of the sawtooth with respect to the ion beam and detector is shown. The shadow cones are indicated, as well as the extra blocking in case of the missing-row and sawtooth reconstructions. lower energies as a result of electronic stopping of protons in the solid. So-called "blocking minima" occur in the surface-peak area because ions backscattered from the second, third, or deeper layers are hindered from leaving the crystal along those directions where other $\mathrm{Ag}$ atoms block their way out.

As is evident from Fig. 2, the missing-row reconstructed surface has the same number of [101] rows exposed to the beam as the unreconstructed surface, though half of them terminate in the second layer. The detector will therefore receive equal backscattering signals from both surfaces in most directions. However, for the reconstructed surface, the signal will be strongly reduced along the [011] exit direction (see Fig. 2); here, strong additional blocking occurs because the backscattering contribution from the nonshadowed atoms in the second layer is obstructed by first-layer atoms. In other directions such as the [123] direction such extra blocking does not occur, since along these the surface atoms are missing. In case of a sawtooth reconstruction, the [101] rows terminate in either the first, second, or third atomic layer (Fig. 2). The amount of additional blocking with respect to the unreconstructed surface is then even larger, and new blocking effects are expected to appear (see below). In the paired-rows model, shadowing and blocking are less effective since the topmost atoms are no longer on lattice sites. Consequently, the backscattering yield in all directions is expected to be higher for this model than for the unreconstructed surface.

These considerations allow a distinction between the different models for the $\mathrm{K}$-induced reconstruction of $\mathrm{Ag}$ (110) from the shape of the blocking patterns alone. Figure 3 shows the measured numbers of Ag layers visi-

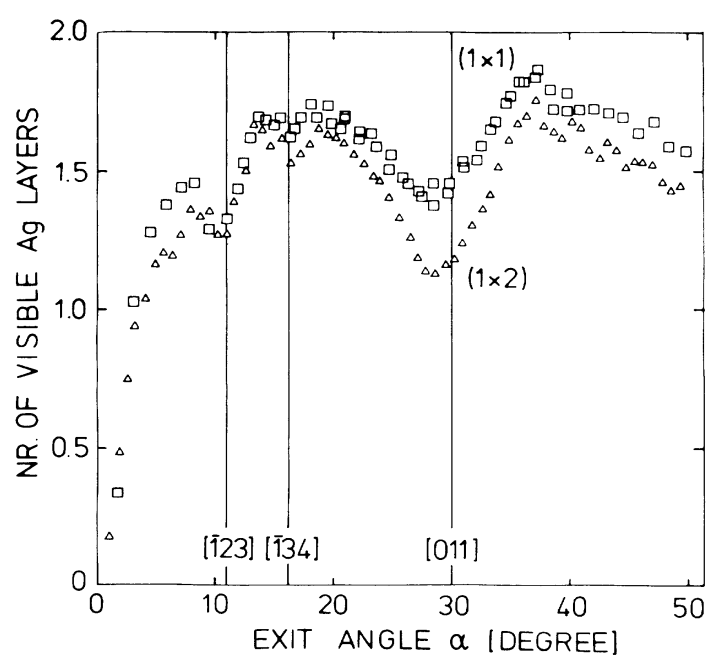

FIG. 3. Blocking patterns measured with $50.6-\mathrm{keV}$ protons in the geometry of Fig. 2 for the clean $\mathrm{Ag}(110)$ surface (squares) and the $(1 \times 2) \mathrm{K}$-induced reconstruction at a $\mathrm{K}$ coverage of 0.39 monolayer (triangles). The vertical lines denote the locations of the [011], [ī34], and [ī23] bulk axes. 
ble to the proton beam and the detector as functions of exit angle $\alpha$ for the clean $(1 \times 1)$ as well as the $\mathrm{K}$-covered $(1 \times 2)$ surface. The fact that for all $\mathrm{K}$ coverages leading to a $(1 \times 2)$ LEED pattern the blocking patterns were identical to within statistical error proves unambiguously that the $(1 \times 2)$ periodicity is caused by reconstruction of the $\mathrm{Ag}$ substrate, and not by the adsorbate overlayer. In addition, it allows us to neglect shadowing and blocking effects of the adsorbed $\mathrm{K}$ atoms in our discussion of the $(1 \times 2)$ blocking pattern.

The depths of the blocking minima in Fig. 3 are consistent with a missing-row reconstruction: The [011] minimum for the reconstructed surface is deeper than for the unreconstructed surface, while the [123] minimum remains unchanged. Also, the backscattering yield changes very little upon reconstruction at those exit angles where no blocking occurs $\left(13^{\circ}<\alpha<20^{\circ}\right.$ and $\alpha>38^{\circ}$ ). We note that the yield at small exit angles, $\alpha<8^{\circ}$, is also lower since at these angles many blocking minima overlap of which half become deeper. In addition, the angular position of the [011] surface-blocking minimum does not coincide with that of the [011] bulk axis. The shift to lower exit angles corresponds to a vertical contraction of the reconstructed surface.

The qualitative interpretation of Fig. 3 in terms of a missing-row-type reconstruction of the $\mathrm{K}$-covered $\mathrm{Ag}$ (110) surface is supported by a comparison of the measured blocking pattern for the $(1 \times 2)$ surface with computer-simulated blocking patterns for the different reconstruction models (Fig. 4). In these Monte Carlo-type simulations ${ }^{17}$ the atomic positions and thermal vibration amplitudes (defined as the onedimensional rms thermal displacements and assumed to be uncorrelated ${ }^{4}$ ) serve as adjustable parameters. In

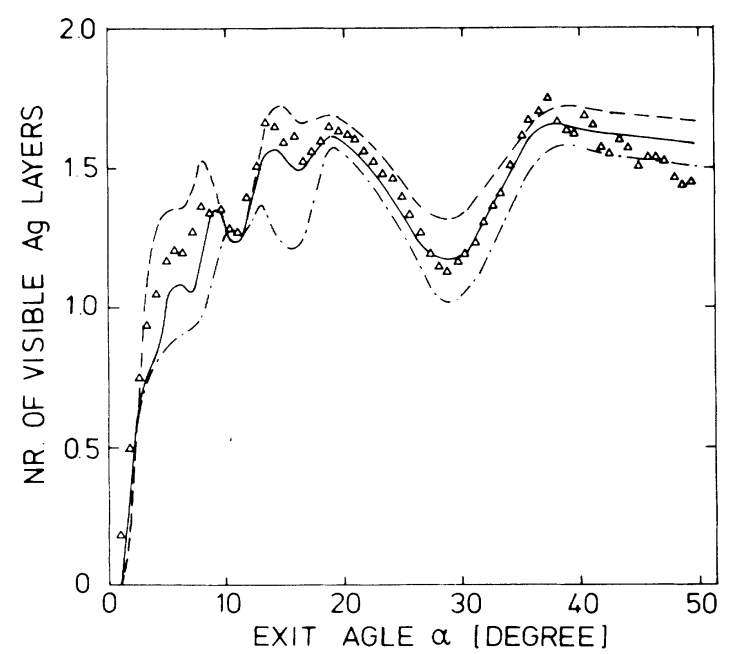

FIG. 4. Comparison of the measured blocking pattern for the $(1 \times 2)$ reconstructed surface with simulated blocking patterns for the missing-row model(solid line), the sawtooth model (dot-dashed line), and the paired-rows model (dashed line). each of the three calculated blocking patterns of Fig. 4 the vibration amplitudes of first-, second-, and deeperlayer $\mathrm{Ag}$ atoms were taken equal to the clean-surface values of $\sigma_{1}=0.15 \AA, \sigma_{2}=0.11 \AA$, and $\sigma_{b}=0.09 \AA$, respectively. The vertical relaxations of first- and secondlayer Ag atoms were chosen such that the calculated blocking minima all appear at the measured blocking angles. Clearly, the simulation result for the missing-row model gives an excellent fit to the experimental data. The amount of blocking predicted for the sawtooth model along the [011] direction is much larger than the observed blocking. In addition, this model predicts, contrary to observations, a substantial deepening of the [134] blocking minimum at an exit angle of $\alpha=16^{\circ}$, resulting from blocking of the signal from the fully exposed third-layer atoms by atoms in the first layer of the sawtooth [Fig. 2 (c)]. The simulation for the pairedrows model was performed by taking the sideways displacements of the surface atoms as small as $0.05 \AA$. The resulting blocking pattern does not show the experimentally observed lowering of the yield in the [011] direction and at exit angles below $8^{\circ}$. Pairing displacements larger than $0.05 \AA$ raise the scattering yield even further above the observed yield.

Additional measurements of surface-blocking patterns in the (110) and the (001) planes, which are perpendicular and parallel to the [110] atom rows, respectively, are also fully supportive of the missing-row model. In the (110) scattering geometry a configuration similar to the one in Fig. 2 results also in a deepening of surfaceblocking minima. In the (001) scattering geometry the measured blocking patterns for the reconstructed and the unreconstructed surfaces are identical to within statistical error, as is expected for the missing-row model since the internal structure of the individual (001) scattering planes is not changed.

Monte Carlo simulations give the best fit for relaxations of $\Delta d_{12} / d=(-9 \pm 2) \%$ and $\Delta d_{23} / d=(-1 \pm 2) \%$ for the first two interlayer distances of the reconstructed surface with respect to the bulk interlayer distance $d$ $(d=1.44 \AA)$. The contraction of the first interlayer distance is equal to that found for clean $\mathrm{Ag}(110)$ to within statistical error. ${ }^{4}$ Remarkably different is the relaxation of the second interlayer distance. For clean $\mathrm{Ag}(110)$, $d_{23}$ is expanded by $(6.0 \pm 2.5) \%{ }^{4}$ Neither inclusion of lateral pairing of [110] rows in the second layer nor inclusion of buckling of the third layer, such as found in recent studies ${ }^{1,2}$ of the missing-row reconstructed Au(110) surface, made the fit to the experimental data better.

The observation of the alkali-metal-induced reconstruction of $\mathrm{Ag}$ (110) lends strong support to an interpretation by Heine and Marks of the Au(110) clean-surface reconstruction in terms of competing forces, exerted on the surface atoms by the metal $s-p$ and $d$ electrons. ${ }^{11}$ In the bulk of a noble metal there is a tension or opposition 
between two types of force: a pairwise repulsion between atoms due to the full $d$ shells, and a multiatom electrongas-mediated attraction due to the $s-p$ electrons and the $s-d$ hybridization. A truncation of the bulk leaves an unstable system which will tend to rearrange under the influence of these forces. At the surface, the $s-p$ electrons are very mobile and can relax normal as well as tangentially to the surface, if the surface is corrugated. A qualitative analysis ${ }^{11}$ of the different contributions to the pseudopotential of Au showed that the $s-p$ electrons, being mobile enough to redistribute, have a tendency to flow into an anomalously attractive region around the atom core. This effect is expected to be strongest in $\mathrm{Au}$ and least pronounced in Ag. ${ }^{11}$ The full $d$ shells which remain essentially unchanged at the surface exert an expansive pressure to balance this contractive stress due to the $s$ - $p$ electrons. This mechanism correctly explains the types of reconstruction observed on the noble-metal surfaces. On (001), there are unfavorably large holes forcing large corrugations in the electron gas. These corrugations are smoothed out by $s$-p-electron flow as discussed above, with the formation of a (111)-type overlayer. ${ }^{18}$ The missing-row reconstruction of the (110) surface corresponds to the formation of a (111) microfacets. The sawtooth reconstruction is obviously unlikely because it contains (111) and (001) facets, the latter being unfavorable from a charge-corrugation point of view.

The Ag(110) surface does not reconstruct because of the smaller attractive part of the $\mathrm{Ag}$ pseudopotential compared with that of Au. However, when extra $s-p$ charge is added, which increases the contractive forces on the surface atoms, a reconstruction into (111) facets is achieved.

It is interesting to review other alkali-metal-induced reconstructions at this stage. In $\mathrm{Pd}$ and $\mathrm{Ni}$ less $s-p$ charge donation should be necessary because of the reduced repulsion of the unfilled $d$ shell; these metals still seem to behave very much like the noble metals, in agreement with theoretical predictions. ${ }^{19}$

This work is sponsored by the Stichting voor Fundamenteel Onderzoek der Materie (FOM) with financial support from the Nederlandse Organisatie voor Zuiver
Wetenschappelijk Onderzoek (ZWO), and is supported by the Deutsche Forschungsgemeinschaft through Sonderforschungsbereich 6 .

\footnotetext{
(a) Present address: Max-Planck-Institut für Strömungsforschung, Bunsenstrasse 10, D-3400 Göttingen, West Germany.
}

(b) Present address: Hahn-Meitner-Institut, Glienicker Strasse 100, D-1000 Berlin 39, West Germany.

${ }^{1}$ M. Copel and T. Gustafsson, Phys. Rev. Lett. 57, 723 (1986)

${ }^{2}$ W. Moritz and D. Wolf, Surf. Sci. 163, L655 (1985), and references therein.

${ }^{3}$ Y. Kuk and L. C. Feldman, Phys. Rev. B 30, 5811 (1984).

${ }^{4}$ E. Holub-Krappe, K. Horn, J. W. M. Frenken, R. L. Krans, and J. F. van der Veen, to be published.

${ }^{5}$ M. Copel, T. Gustafsson, W. R. Graham, and S. M. Yalisove, Phys. Rev. B 33, 8110 (1986).

${ }^{6}$ C. J. Barnes, M. Q. Ding, M. Lindroos, and D. A. King, Surf. Sci. 162, 59 (1985).

${ }^{7}$ B. E. Hayden, K. C. Prince, P. J. Davie, G. Paolucci, and A. M. Bradshaw, Solid State Commun. 48, 325 (1983).

${ }^{8}$ S. M. Francis and N. V. Richardson, Surf. Sci. 152/153, 63 (1985)

${ }^{9}$ M. Copel, W. R. Graham, T. Gustafsson, and S. M. Yalisove, Solid State Commun. 54, 695 (1985).

${ }^{10}$ R. J. Behm, G. Ertl, D. K. Flynn, K. D. Jamison, and P. A. Thiel, to be published.

${ }^{11}$ V. Heine and L. D. Marks, Surf. Sci. 165, 65 (1986).

${ }^{12}$ P. J. Goddard, J. West, and R. M. Lambert, Surf. Sci. 71, 447 (1978), and references therein.

${ }^{13}$ T. Engel and K. H. Rieder, in Structural Studies of Surfaces, edited by G. Höhler, Springer Tracts in Modern Physics Vol. 91 (Springer-Verlag, Berlin, 1982).

${ }^{14}$ A. M. Lahee, W. Allison, R. F. Willis, and K. H. Rieder, Surf. Sci. 126, 654 (1983).

${ }^{15}$ H. P. Bonzel and S. Ferrer, Surf. Sci. 118, L263 (1982)

${ }^{16}$ J. F. van der Veen, Surf. Sci. Rep. 5, 199 (1985).

${ }^{17}$ J. W. M. Frenken, R. M. Tromp, and J. F. van der Veen, Nucl. Instrum. Methods Phys. Res., Sect. B 17, 334 (1986).

${ }^{18}$ M. A. van Hove, R. J. Koestner, P. C. Stair, J. P. Bibérian, L. L. Kesmodel, I. Bartoš, and G. A. Somorjai, Surf. Sci. 103, 189, 218 (1981).

${ }^{19}$ D. G. Pettifor, J. Phys. F 8, 219 (1978). 